\title{
Investigation of the ion dose non-uniformity caused by sheath-lens focusing effect on
} silicon wafers

\author{
Holtzer, N.; Stamate, Eugen; Toyoda, H.; Sugai, H.
}

Published in:

Thin Solid Films

Link to article, DOI:

10.1016/j.tsf.2006.10.099

Publication date:

2007

Document Version

Publisher's PDF, also known as Version of record

Link back to DTU Orbit

Citation (APA):

Holtzer, N., Stamate, E., Toyoda, H., \& Sugai, H. (2007). Investigation of the ion dose non-uniformity caused by sheath-lens focusing effect on silicon wafers. Thin Solid Films, 515, 4887-4891.

https://doi.org/10.1016/j.tsf.2006.10.099

\section{General rights}

Copyright and moral rights for the publications made accessible in the public portal are retained by the authors and/or other copyright owners and it is a condition of accessing publications that users recognise and abide by the legal requirements associated with these rights.

- Users may download and print one copy of any publication from the public portal for the purpose of private study or research.

- You may not further distribute the material or use it for any profit-making activity or commercial gain

- You may freely distribute the URL identifying the publication in the public portal 


\title{
Investigation of the ion dose non-uniformity caused by sheath-lens focusing effect on silicon wafers
}

\author{
N. Holtzer *, E. Stamate ${ }^{1}$, H. Toyoda, H. Sugai \\ Department of Electrical Engineering and Computer Science, Nagoya University, Furo-cho, Chikusa-ku, Nagoya 464-8603, Japan
}

Available online 11 December 2006

\begin{abstract}
The ion dose non-uniformity induced on the wafer surface by modal and discrete focusing effects is investigated for different plasma densities and implantation parameters. Measured impact radius agrees well with values obtained by simulation. The optical pattern observed on the wafer surface is correlated with the ion dose by FT-IR measurements. The applicability of a previously proposed vertical ring is demonstrated, the ring being able to considerably improve the ion flux uniformity by shifting the discrete focusing effect out of the wafer surface and reducing the modal focusing. Experiments are performed in an inductively coupled plasma produced in hydrogen.

(C) 2006 Elsevier B.V. All rights reserved.
\end{abstract}

Keywords: Plasma immersion ion implantation; Dose uniformity; Sheath-lens focusing effect; Guard ring

\section{Introduction}

Ion implantation is a widely used process in large area doping of semiconductor wafers and patterned structures [1-3] and improvement of wear and corrosion behavior of metal surfaces [4,5]. Recent applications also include biomedical technologies [6] and arc ion plating [7]. Plasma Immersion Ion Implantation (PIII), otherwise called Plasma Source Ion Implantation (PSII), has several advantages over conventional ion beam implantation including higher ion current densities and a wider ion energy range, making it possible to treat targets of complex shapes in a rather short time [8]. In PIII the substrate is immersed in the plasma and biased negatively with highvoltage pulses from hundreds of volts up to $100 \mathrm{kV}$. Consequently, a large sheath forms the shape of which is strongly dependent on the substrate geometry and also on the electrical properties of adjacent holders. As a result, the sheath is a two or three dimensional structure in which potential distribution determines the ion motion until reaching the surface. Over the years, significant efforts were devoted to predict and improve the ion flux over the treated surface [9-12].

\footnotetext{
* Corresponding author.

E-mail address: nico_h@nuee.nagoya-u.ac.jp (N. Holtzer).

${ }^{1}$ Permanent address: Risø National Laboratory, Optics and Plasma Research Department, DK-4000, Roskilde, Denmark.
}

Ion dose non-uniformities in PIII of silicon wafers have been reported since 1998 [13] but the phenomenology inducing the observed pattern was explained very recently. Thus, it was demonstrated that the modal [14] and discrete [15] focusing effects are responsible for the formation of a passive surface (no ion impact) and the presence of an intense focusing ring that significantly degraded the ion flux uniformity [16]. A solution to improve the ion flux uniformity by introducing a vertical ring (VR) was actually proposed $[17,18]$ but its effectiveness was not yet validated on real PIII conditions.

In this work, we are investigating the influence of plasma density and implantation parameters on the ion dose nonuniformity induced by modal and discrete focusing effects. The VR ability to shift the discrete focusing from the wafer surface is also verified. FT-IR measurements are used to correlate the optical pattern observed on the wafer surface with ion flux predicted by simulations.

\section{Experimental setup}

Experiments are realized in a $13.56 \mathrm{MHz}$ driven ICP reactor $\left(35 \times 35 \times 35 \mathrm{~cm}^{3}\right)$ that is shown schematically in Fig. $1 . \mathrm{H}_{2}$ plasma density, ranging from $7 \times 10^{14} \mathrm{~m}^{-3}$ up to $7 \times 10^{16} \mathrm{~m}^{-3}$, was monitored by a plasma absorption probe (PAP) connected to a high speed Network Analyzer [10]. Silicon wafers of $100 \mathrm{~mm}$ in diameter have been biased from $-5 \mathrm{kV}$ down to 


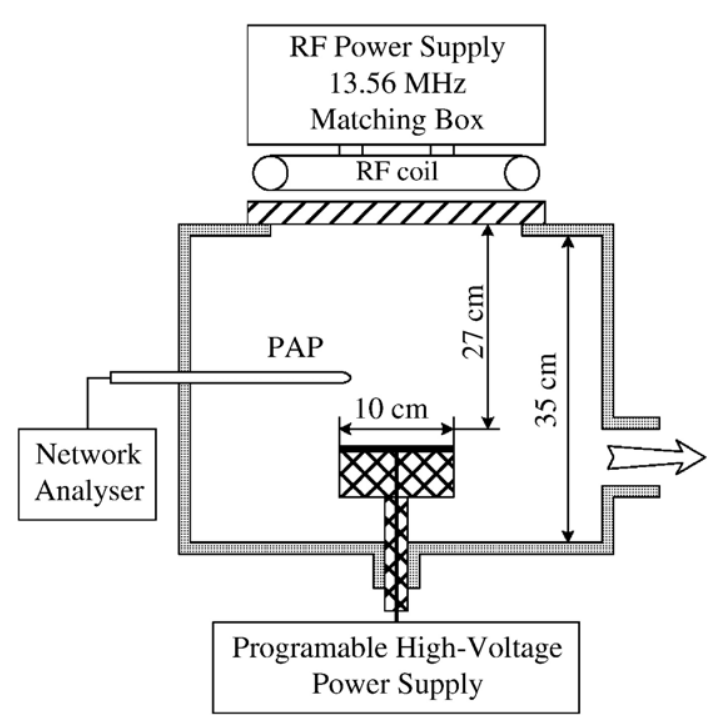

Fig. 1. Schematic diagram of the ICP reactor.

$-10 \mathrm{kV}$ at a fixed pulse width of $50 \mu \mathrm{s}$, and a pulse frequency of $20 \mathrm{~Hz}$.

The wafers were fixed to a multi-purpose insulated stand placed $27 \mathrm{~cm}$ below the dielectric window as shown in Fig. 2, where (a) corresponds to a wafer with no additional ring, (b) a wafer with guard ring, and (c) a wafer with vertical ring (VR), respectively. The VR resembles the lateral surface of a $48 \mathrm{~mm}$ tall cylinder surrounding the wafer stand. A small gap of about $2 \mathrm{~mm}$ was set between the wafer edge and the VR that has been electrically connected to the same bias as the wafer by additional wiring inside the stand.

\section{Results and discussions}

\subsection{Dependence of discrete focusing on plasma density and implantation parameters}

When applying a negative pulse to the wafer, the lighter electrons are repelled within a time interval correlated with electron plasma frequency leaving behind the ions that respond much slower due to their heavier mass. This initial charge dynamics results in the formation of what is defined as a matrix sheath [8]. After, the ions start being accelerated and the sheath expands, reaching a steady state in less than $5 \mu \mathrm{s}$ [8]. Since this time is less than $10 \%$ of the pulse width, we can assume that the sheath is stationary after its formation. In the case of a wafer with no surrounding electrodes (see Fig. 2(a)) the sheath expands not only in the $z$ direction (defined as perpendicular to the wafer surface) but also behind the wafer plane $(-z)$, resulting in the formation of both modal and discrete focusing effects [14,15]. In order to obtain the potential distribution in the sheath, we solved the Poisson equation in three dimensions using the finite element method [16]. Since the applied pulsed bias, $V_{\mathrm{o}}$, was very large with respect to plasma potential the sheath edge surface $\Sigma$ was decided as $V=2 \%$ of $V_{\mathrm{o}}$. The sheath edge profiles, $\Sigma_{(1-5)}$, for different plasma densities are shown in Fig. 3, where the applied bias was kept constant to $V_{\mathrm{o}}=-10 \mathrm{kV}$ and the wafer radius, $R_{\mathrm{o}}$,

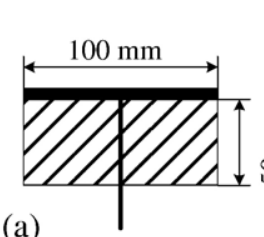

(a)

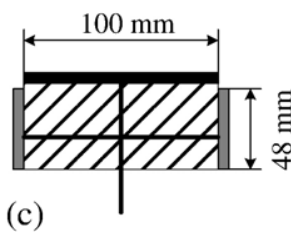

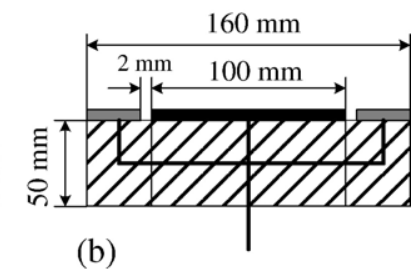

(b)

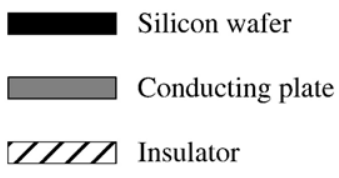

Fig. 2. Different geometries for the wafer stand; (a) wafer with no additional ring, (b) wafer with guard ring, and (c) wafer with vertical ring.

was set to $50 \mathrm{~mm}$. The plasma densities, $n_{\mathrm{e}}$, used for this simulation are presented later on in Fig. 7. Let us consider that ions are entering $\Sigma$ with coordinates $\left(x_{\mathrm{o}}, y_{\mathrm{o}}, z_{\mathrm{o}}\right)$ and velocities $\left(v_{\mathrm{xo}}, v_{\mathrm{yo}}, v_{\mathrm{zo}}\right)$ reaching the wafer surface at $\left(x_{\mathrm{f}}, y_{\mathrm{f}}, 0\right)$. We can also separate all ions entering the sheath in frontal ions for $z_{\mathrm{o}} \geq 0$ and backside ions for $z_{\mathrm{o}}<0$. From previous calculations on ion trajectories it was shown that the passive surface was delineated close to the wafer edge by a ring of intense ion flux that resulted from discrete focusing. Because the backside ions are mostly directed to the central part of the wafer the inner contour of the passive surface is delineated by those ions that enter the sheath with $v_{\mathrm{zo}}=0$, a location where the component of the electric field in $z$ direction changes its sign. The trajectories of test ions entering each $\Sigma_{(1-5)}$ with $v_{\mathrm{zo}}=0$ are also shown in Fig. 3 . The radius delineating the inner diameter of the passive surface was defined as impact radius, $r_{\text {imp. }}$. As expected, $r_{\text {imp }}$ increases as the sheath thickness shrinks by increasing $n_{\mathrm{e}}$. The passive surface corresponds to $R_{\mathrm{o}} \geq r>r_{\text {imp }}$ while for $r \leq r_{\text {imp }}$ the ion flux includes the contribution of both discrete and modal focusing. For instance, the impact locations of 3500 test ions entering the sheath obtained for $n_{\mathrm{e}}=5 \times 10^{15} \mathrm{~m}^{-3}, T_{\mathrm{e}}=3 \mathrm{eV}$ and $V_{\mathrm{o}}=-10 \mathrm{kV}$ are shown in Fig. 4(a) for $z_{\mathrm{o}} \geq 0$ and (b) $z_{\mathrm{o}}<0$. If focusing of frontal ions lead to the formation of a passive surface and high dose ring through discrete focusing, ions coming from the back

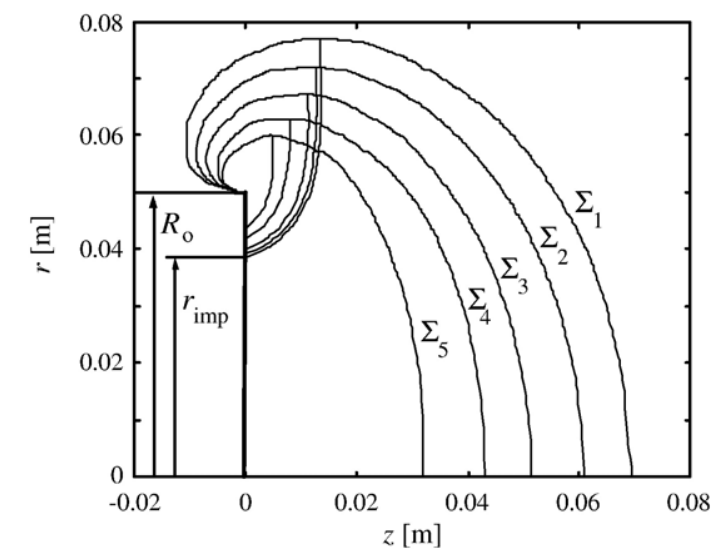

Fig. 3. Sheath edge profiles, $\Sigma$, and ion trajectories with $v_{\mathrm{zo}}=0$ for $V_{\mathrm{o}}=-10 \mathrm{kV}$ and plasma densities presented in Fig. 7. 
(a)

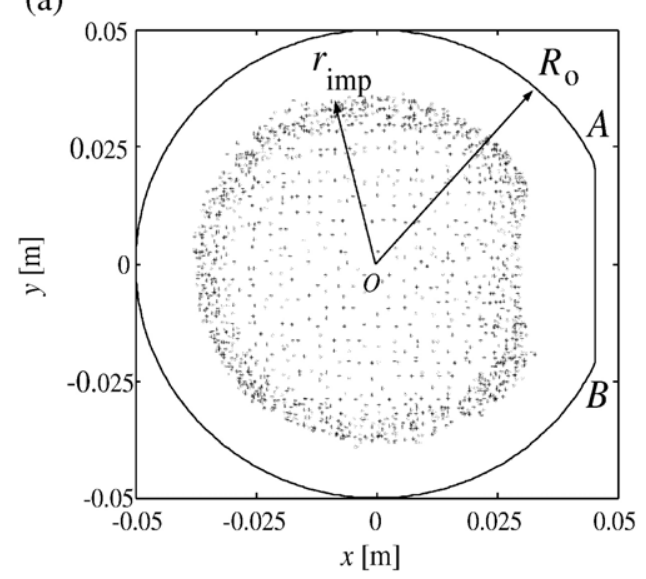

(b)

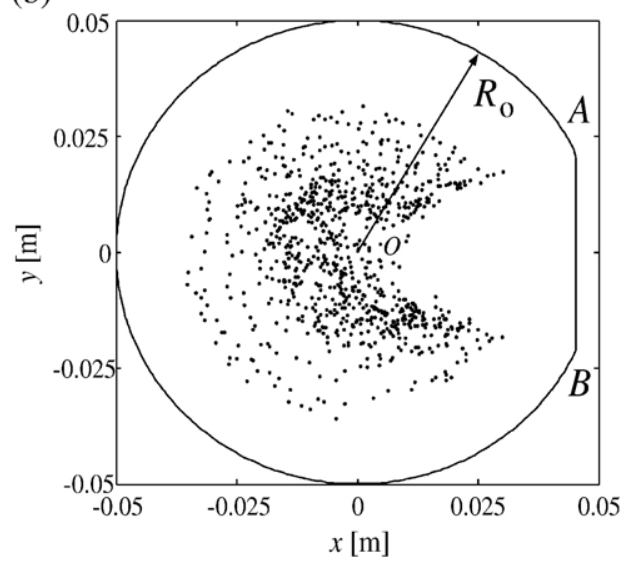

Fig. 4. Impact locations of 3500 test ions entering the sheath obtained for $n_{\mathrm{e}}=5 \times 10^{15} \mathrm{~m}^{-3}, T_{\mathrm{e}}=3 \mathrm{eV}$ and $V_{\mathrm{o}}=-10 \mathrm{kV}$, (a) for $z_{\mathrm{o}} \geq 0$ (discrete focusing) and (b) $z_{\mathrm{o}}<0$ (modal focusing).

side are concentrated around the wafer center, with a clear shadow induced by the wafer flat (line AB in Fig. 4) that has limited the sheath expansion in $-\mathrm{z}$ direction because the stand followed a circular profile.

The dependence of $r_{\text {imp }}$ on plasma parameters for a dc bias was already reported for disk and square electrodes of about $10 \mathrm{~mm}$, a size much smaller than the plasma chamber $(25 \mathrm{~cm}$ in diameter) [17]. In present work, and PIII in general, the sheath size is just a few times smaller or even comparable with the

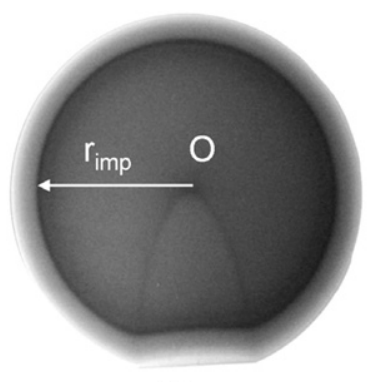

(a)

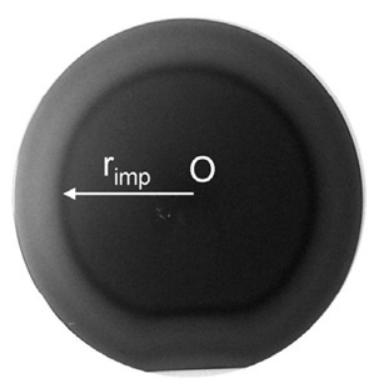

(b)
Fig. 5. Photographs of two wafers treated at $p=35$ mTorr, where (a) $n_{\mathrm{e}}=5 \times 10^{16} \mathrm{~m}^{-3}, V_{\mathrm{o}}=-8 \mathrm{kV}$ and (b) $n_{\mathrm{e}}=8 \times 10^{14} \mathrm{~m}^{-3}, V_{\mathrm{o}}=-10 \mathrm{kV}$.

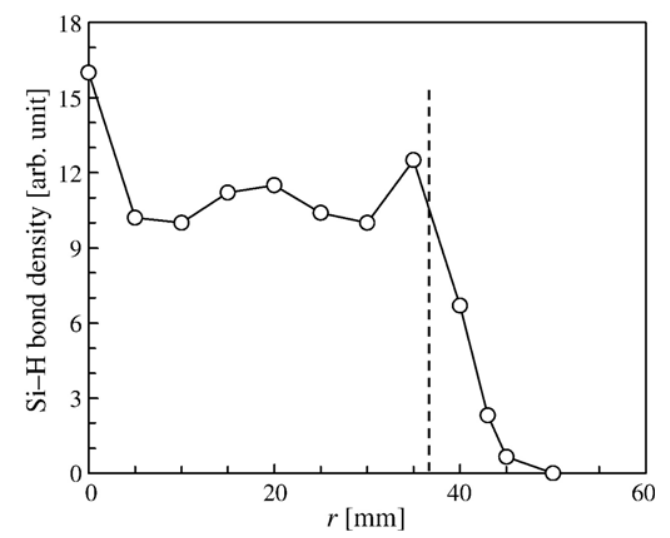

Fig. 6. FT-IR measurements of the $\mathrm{Si}-\mathrm{H}$ bond density in the radial direction for a wafer that exhibited after treatment a $r_{\text {imp }}=3.7 \mathrm{~mm}$ marked with dashed line.

reactor size or only a few times smaller, and it is therefore important to verify the influence of plasma density and implantation parameters on discrete and modal focusing effects. As an example the photographs of two wafers treated at $p=35 \mathrm{mTorr}$ are shown in Fig. 5 where (a) $n_{\mathrm{e}}=5 \times 10^{16} \mathrm{~m}^{-3}, V_{\mathrm{o}}=-8 \mathrm{kV}$ and (b) $n_{\mathrm{e}}=8 \times 10^{14} \mathrm{~m}^{-3}, V_{\mathrm{o}}=-10 \mathrm{kV}$, respectively. The passive surface delineated by discrete focusing effect increased for lower plasma density because of the sheath expansion. The shadow predicted by simulations from Fig. 3 is also visible in Fig. 5(a). To prove that the optical pattern is correlated with surface modification by ion implantation, we performed FT-IR measurements of the $\mathrm{Si}-\mathrm{H}$ bond density in the radial direction of a wafer that exhibited a $r_{\text {imp }}=3.7 \mathrm{~mm}$ after treatment. The FT-IR result is shown in Fig. 6 where one can see that the passive surface corresponded, as expected, to a very low $\mathrm{Si}-\mathrm{H}$ bond density and that the high ion dose induced by discrete focusing resulted in a peak just around the measured $r_{\text {imp }}$. The highest $\mathrm{Si}-$ $\mathrm{H}$ bond density was measured at the wafer center where the frontal ions reach the surface at normal incidence while the backside ions reach it at oblique incidence.

In order to crosscheck the simulation with experiments we treated 5 wafers for $p=35$ mTorr and a fixed bias of $-8 \mathrm{kV}$ for different $n_{\mathrm{e}}$ and measured the $r_{\text {imp. }}$. Then after we used the

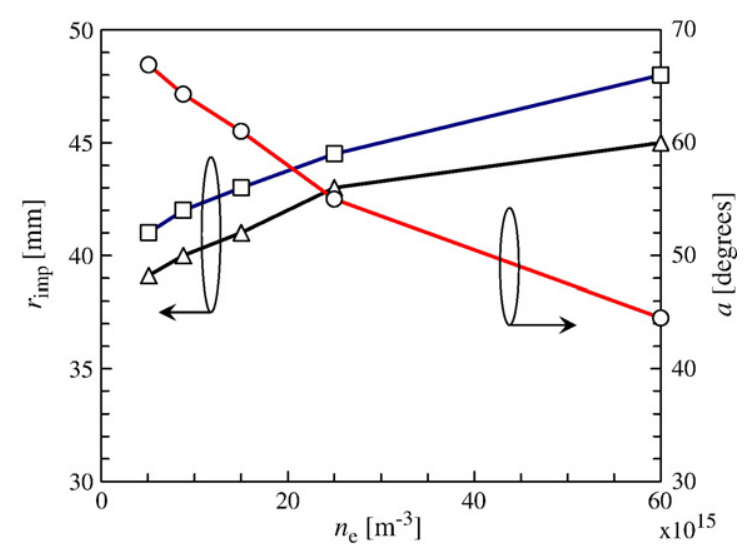

Fig. 7. Experimental $r_{\text {imp }}$ (open triangles) for different $n_{\mathrm{e}}$ where $V_{\mathrm{o}}=-8 \mathrm{kV}$ and $p=35$ mTorr. The values of $r_{\text {imp }}$ by simulations (open square) and corresponding impact angles, $\alpha$ (open circles), are also included. 


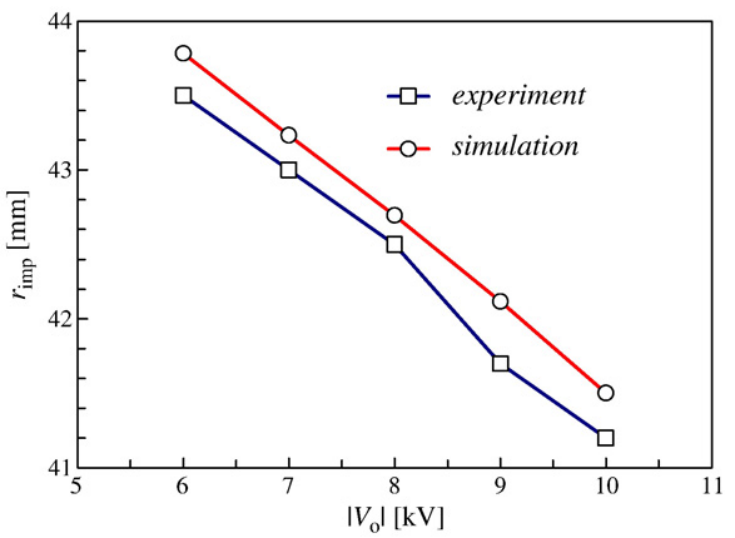

Fig. 8. Experimental $r_{\text {imp }}$ with open squares and simulation values with open circles for different $\left|V_{\mathrm{o}}\right|$, where $n_{\mathrm{e}}=6 \times 10^{15} \mathrm{~m}^{-3}$ and $T_{\mathrm{e}}=3.5 \mathrm{eV}$.

experimental values of $n_{\mathrm{e}}, T_{\mathrm{e}}$ and $V_{\mathrm{o}}$ as input parameters for simulation and computed the corresponding values for $r_{\text {imp. This }}$ result is shown in Fig. 7 where experimental $r_{\text {imp }}$ is shown with open circles and theoretical values with open squares. The agreement is quite good if we take into consideration that $n_{\mathrm{e}}$ is not uniform in both $z$ and radial directions (less than $25 \%$ within the range of $\sum$ shown in Fig. 3) a fact that could not be accounted in our simulation. The impact angle, $\alpha$, is an important parameter for ion induced surface modification because the implantation depth and/or sputtering yield depends on it. Most ions forming the high dose ring reach the surface with a narrow range of $\alpha$. The $\alpha$ of a test ion entering the sheath with $v_{\mathrm{zo}}=0$ for different $n_{\mathrm{e}}$ is also shown in Fig. 7 where $\alpha$ is defined as $\alpha=0^{\circ}$ for normal incidence. As expected $\alpha$ decreases as the sheath gets thinner, but it remains larger than $40^{\circ}$. The sheath also expands with an increasing $V_{\mathrm{o}}$, not only by a decreasing $n_{\mathrm{e}}$. Thus, experimental $r_{\text {imp }}$ with squares and simulation values with circles are shown Fig. 8, for different $\left|V_{\mathrm{o}}\right|$, where $n_{\mathrm{e}}=6 \times 10^{15} \mathrm{~m}^{-3}$ and $T_{\mathrm{e}}=3.5 \mathrm{eV}$. The agreement is fair and demonstrates that one can predict with a certain accuracy the shift of the discrete focusing with $n_{\mathrm{e}}$ and $V_{\mathrm{o}}$ using the assumption of a dc sheath. The sheath thickness depends weakly on $T_{\mathrm{e}}$ thus it was not considered in the present work.

\subsection{Improvement of ion dose uniformity using a vertical ring}

Increase of ion current, $I_{\mathrm{i}}$, of a planar probe as a result of sheath expansion by decreasing the bias with respect to plasma potential, $V_{\mathrm{pl}}$, was known since the pioneering work of Langmuir [19]. Due to lack of appropriate analytic models to describe $I_{\mathrm{i}}\left(V_{\mathrm{o}}\right)$ Medicus proposed a guard-ring-probe [20] able to reduce the increasing $I_{\mathrm{i}}$ by improving the parallelism of the sheath front with the probe surface. The same solution but for a larger scale was suggested to avoid the ion dose non-uniformity observed in PIII of silicon wafers [21]. However simulations showed that such a guard ring cannot prevent the back side ions to reach the probe (wafer) surface the modal focusing effect and that the discrete focusing effect is still taking place on the guard ring [17]. In order to prove this for PIII experiments, we used the stage setup presented in Fig. 2(b) where a guard ring of $160 \mathrm{~mm}$ in external diameter was used to surround the wafer

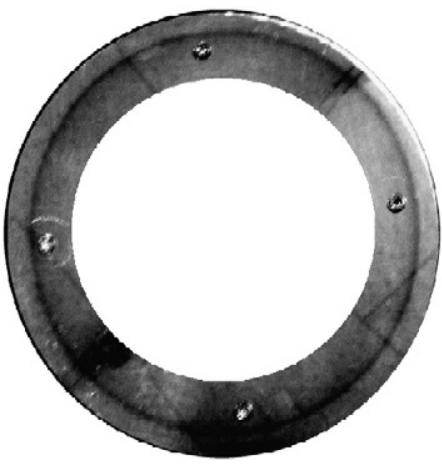

Fig. 9. Photograph of a guard ring after having been treated for an effective time of $15 \mathrm{~s}$ for $p=35 \mathrm{mTorr}, n_{\mathrm{e}}=6 \times 10^{15} \mathrm{~m}^{-3}, T_{\mathrm{e}}=3.9 \mathrm{eV}$ and $V_{\mathrm{o}}=-8 \mathrm{kV}$.

stand with a gap interval of $1 \mathrm{~mm}$. The photograph of the guard ring after treatment for an effective time of $15 \mathrm{~s}$ is shown in Fig. 9 where $p=35$ mTorr, $n_{\mathrm{e}}=6 \times 10^{15} \mathrm{~m}^{-3}, T_{\mathrm{e}}=3.9 \mathrm{eV}$ and $V_{\mathrm{o}}=-8 \mathrm{kV}$. One can very well distinguish a darker ring formed concentric with the guard-ring structure by discretely focused ions at about $7 \mathrm{~mm}$ from the external edge. As discussed in Figs. 7 and 8, the location of this ring will shift depending on the sheath thickness, making it actually possible to reach the wafer surface if the sheath exceeds a certain size.

As an alternative solution to the guard ring a vertical ring was proposed but not verified in real PIII situation [17]. In order to do so we used the arrangement presented in Fig. 2(c) where the vertical side of the stand was covered by a $48 \mathrm{~mm}$ tall metallic cylinder that was electrically connected to the same $V_{\mathrm{o}}$ as the wafer. The photographs of two wafers treated for identical plasma and implantation parameters $\left(n_{\mathrm{e}}=1.7 \times 10^{16} \mathrm{~m}^{-3}\right.$, $T_{\mathrm{e}}=4 \mathrm{eV}, p=35$ mTorr $V_{\mathrm{o}}=-8 \mathrm{kV}$ ) are shown in Fig. 10 where (a) is without VR and (b) with VR. While wafer in Fig. 10 (a) exhibits both the modal and discrete focusing, the wafer in Fig. 10(b) shows no evidence of any non-uniformity induced by ion focusing. Moreover, the FT-IR measurement on the wafer with VR showed a constant level for $\mathrm{Si}-\mathrm{H}$ bond in the radial direction. Thus besides improving the ion flux uniformity the VR provides some additional advantages such as the use of a plasma chamber with lower diameter and a power supply of less current if height of VR is optimized to a minimum value for which the discrete focusing is not shifting on the wafer surface.

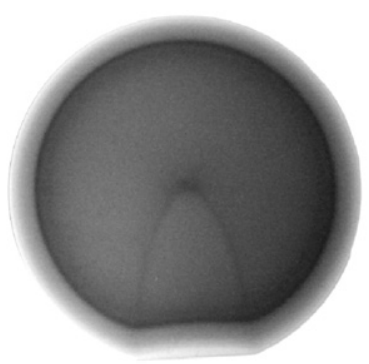

(a)

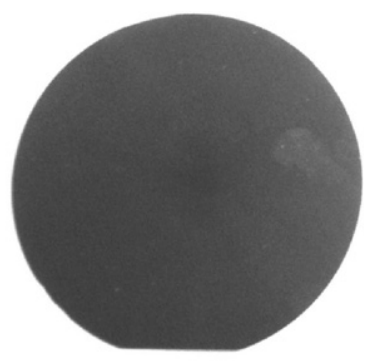

(b)
Fig. 10. Photographs of two wafers treated for $n_{\mathrm{e}}=1.7 \times 10^{16} \mathrm{~m}^{-3}, T_{\mathrm{e}}=4 \mathrm{eV}$, $p=35$ mTorr and $V_{\mathrm{o}}=-8 \mathrm{kV}$, where (a) was without vertical ring and (b) with vertical ring 


\section{Conclusions}

The ion dose non-uniformity induced on the wafer surface by modal and discrete focusing effects was investigated for different plasma densities and implantation parameters. The measured $r_{\text {imp }}$ agreed well with values obtained by simulation. The optical pattern on the wafer surface was correlated with the ion dose by FT-IR measurements. The applicability of a previously proposed vertical ring was demonstrated, the ring has been able to considerably improve the ion flux uniformity by shifting the discrete focusing effect out of the wafer surface and reducing the modal focusing.

\section{Acknowledgements}

This work has been partially supported by the "PlasmaNano" Center of Excellence Project of MEXT, Japan. One of the authors (N. H.) acknowledges financial support from the French Ministry of Foreign Affairs through the "Lavoisier" fellowship.

\section{References}

[1] P.K. Chu, S. Quin, C. Chan, N.W. Cheung, L.A. Larson, Mater. Sci. Eng., R Rep. 17 (1996) 207.

[2] A. Anders, Handbook of Plasma Immersion Ion Implantation and Deposition, Wiley, New York, 2000.
[3] N.W. Cheung, Nucl. Instrum. Methods B 55 (1991) 811.

[4] J.R. Conrad, J.L. Radtke, R.A. Dodd, F.J. Worzala, N.C. Tran, J. Appl. Phys. 62 (1987) 4591.

[5] K.C. Walter, J. Vac. Sci. Technol., B 30 (1994) 945.

[6] P.K. Chu, Nucl. Instrum. Methods B 242 (2006) 1.

[7] A. Andres, S. Andres, I.G. Brown, M.R. Dickinson, R.A. MacGill, J. Vac. Sci. Technol., B 815 (1994).

[8] J. Pelletier, A. Anders, IEEE Trans. Plasma Sci. 33 (2005) 1944.

[9] J.R. Conrad, J. Appl. Phys. 62 (1987) 777.

[10] I.J. Donnelly, P.A. Watterson, J. Phys. D: Appl. Phys. 22 (1989) 90.

[11] T.E. Sheridan, Appl. Phys. Lett. 68 (1996) 1918.

[12] D. Kim, D. Economou, J. Appl. Phys. 94 (2003) 2852.

[13] Z. Fan, P.K. Chu, C. Chan, N.W. Cheung, Appl. Phys. Lett. 73 (1998) 202.

[14] E. Stamate, H. Sugai, Phys. Rev. Lett. 94 (2005) 125004.

[15] E. Stamate, H. Sugai, Phys. Rev. E. 72 (2005) 036407.

[16] E. Stamate, N. Holtzer, H. Sugai, Appl. Phys. Lett. 86 (2005) 261501.

[17] E. Stamate, N. Holtzer, H. Sugai, Thin Solid Films 506-507 (2006) 571.

[18] E. Stamate, H. Sugai, Thin Solid Films (this TSF volume).

[19] I. Langmuir, Phys. Rev. 33 (1929) 954.

[20] G. Medicus, J. Appl. Phys. 37 (1966) 215.

[21] X.B. Tian, P.K. Chu, Appl. Phys. Lett. 81 (2002) 3744. 